\title{
ASSESSMENT OF INFLAMMATORY MARKERS AND LIPID PROFILE LEVELS IN NORMOTENSIVE, PRIMARY HYPERTENSIVE AND SECONDARY HYPERTENSIVE MALE OBESE INDIVIDUALS
}

\author{
Srinivasa Ravikiran Suri ${ }^{1}$, Abdul Raoof Omer Siddiqui², Manikanta $M^{3}$ \\ ${ }^{1}$ Assistant Professor, Department of Physiology, Shadan Institute of Medical Sciences. \\ ${ }^{2}$ Assistant Professor, Department of Physiology, Shadan Institute of Medical Sciences. \\ ${ }^{3}$ Tutor, Department of Pharmacology, Shadan Institute of Medical Sciences.
}

\section{ABSTRACT}

\section{BACKGROUND}

Hypertension is recognized as the most common cardiovascular disorder and a leading cause of morbidity and mortality in both developed and developing countries. Primary hypertension is an indicator for further complications. Hyperlipidaemia is an indicator for hypertension and recognized as independent risk factor for cardiovascular disease.

\section{OBJECTIVE}

To assess the C-reactive protein, erythrocyte sedimentation rate and plasma lipid profile in primary and secondary hypertensive and normotensive subjects.

\section{MATERIALS AND METHODS}

In this case-control observational study the ESR, CRP and lipid profile (Twelve hour fasting lipid analysis was done for Serum triglycerides (TG), total cholesterol (TC), High Density Lipoprotein (HDL), Low Density Lipoprotein (LDL) test values were compared in 150 members. Height and weight were measured with the subject in light clothes without shoes and Body Mass Index (BMI) $(\mathrm{Kg} / \mathrm{m} 2)$ was calculated. Statistical analysis was done by Microsoft office 2010. Predefined inclusion and exclusion criteria were set for selection of the study group.

\section{RESULTS}

We observed increase in ESR, triglycerides, cholesterol and LDL-cholesterol levels in secondary hypertensive people as compared with normotensive and primary hypertensive individuals. HDL cholesterol levels are decreased in secondary hypertensive male individuals, but no change is observed in HDL cholesterol levels in primary hypertensive male. We emphasized minimal increase in CRP levels in secondary hypertensive male obese individuals as compared with normotensive individuals.

\section{CONCLUSION}

Increase in ESR, triglycerides, cholesterol and LDL-C indicates person is prone to subacute infection and inflammation. He should be thoroughly examined. Change in lipid profile and increase in ESR levels may lead to further cardiovascular disorders.

\section{KEYWORDS}

CRP, ESR, Lipid Profile.

HOW TO CITE THIS ARTICLE: Srinivasa Ravikiran Suri, Abdul Raoof Omer Siddiqui, Manikanta M. "Assessment of Inflammatory Markers and Lipid Profile Levels in Normotensive, Primary Hypertensive and Secondary Hypertensive Male Obese Individuals." Journal of Evolution of Medical and Dental Sciences 2015; Vol. 4, Issue 98, December 07; Page: 16337-16340, DOI: $10.14260 /$ jemds $/ 2015 / 2413$

\section{INTRODUCTION}

Blood pressure is a primary indicator for further disorders. Recently published data also suggests that chronic inflammation could be an independent risk factor for high blood pressure. C-reactive protein has been shown to be associated to high blood pressure in a few well-controlled studies. ${ }^{1}$ Several prospective cohort studies have shown that acute systemic inflammation is associated with an increased risk of acute cardiovascular events and cardiovascular mortality. ${ }^{2}$

Financial or Other, Competing Interest: None.

Submission 20-11-2015, Peer Review 21-11-2015,

Acceptance 01-12-2015, Published 05-12-2015.

Corresponding Author:

Srinivasa Ravikiran Suri,

8-19/1, Sairam enclave,

Near SMP Model School, Hydershakote,

Rajendra Nagar Mandal,

Hyderabad-500009. Telangana.

E-mail: ssphysiology@gmail.com

DOI:10.14260/jemds/2015/2413
Several previous studies showed the relation between hyperlipidemia and hypertension. ${ }^{3}$

The Erythrocyte Sedimentation Rate (ESR) is a nonspecific test that is often used as an indicator of active diseases of inflammation. ${ }^{4}$ Statins lower C-Reactive Protein (CRP) values, suggesting that some of their protective effects may be mediated through suppression of inflammation or cytokines.

Some suggest that lipid lowering agents, Angiotensin Converting Enzyme (ACE) inhibitors, Angiotensin Receptor Blockers (ARBs), anti-diabetic agents, anti-inflammatory and antiplatelet agents, vitamin $\mathrm{E}$ and beta-adrenoreceptors, antagonistic lower serum levels of CRP, while vitamin C, oral estrogen and hydrochlorothiazide do not effect CRP levels. ${ }^{5}$ CRP a marker for systemic inflammation predicts the risk of myocardial infarction and stroke. Reduction in incidence of $1^{\text {st }}$ myocardial infarction associated with the use of aspirin appears to be directly related to the levels of CRP. ${ }^{6}$ 
If there is any excessive intake of cholesterol, saturated fats and other sources of calories and subsequent disturbance of lipid profile leads to increased triglycerides and cholesterol levels and they are associated with obesity and subsequently hypertension. ${ }^{7}$ Some studies also showed a significant relationship between Blood Pressure (BP) and intracellular adhesion molecule- 1 and interleukin- 6 levels and suggested that elevated BP increases the risk of atherosclerosis by promoting inflammatory activation of the arterial wall. ${ }^{8}$ Uncontrolled hypertension can cause damage to all organs of the body. ${ }^{9}$ Age and gender differences also affect serum lipid levels considerably. ${ }^{10,11}$

Atherosclerosis is most extensive and severe in hypertensive persons than in normotensive. ${ }^{12}$ CRP levels were associated with raised serum fibrinogen, sialic acid, total cholesterol, triglycerides and glucose and apolipoprotein B values. CRP levels were negatively associated with high density lipoprotein cholesterol (HDL-C).

Among the older age individuals many environmental lifestyle risk factors for cardiovascular disease are associated with raised CRP levels. CRP functions as atherosclerotic factor as well as powerful risk marker. ${ }^{13}$ Decreased serum magnesium levels have been documented in people with obesity, in those who are also having elevated CRP levels. Individuals with intake of magnesium below the recommended daily allowance are most likely to have elevated CRP, which may contribute to cardiovascular diseases. ${ }^{14}$

Low fat diets that are high in carbohydrates have a tendency to increase fasting triglycerides level, acute fat ingestion also raises postprandial triglycerides, particularly when carbohydrate intake is high. ${ }^{15} \mathrm{HDL}$-cholesterol levels are seen due to low fat diet and there is a relation between low fat diet and benefits of decreased total plasma cholesterol levels and reduction in body weight. 16,17

\section{MATERIALS AND METHODS}

We designed this study to find out primary hypertension and secondary hypertension are major risk factors for future disorders. This study was conducted at Shadan Institute of Medical Sciences and Teaching Hospital, Hyderabad. Participants were obese employees and industrial workers. Mode of selection was made based on their health condition. We excluded chronic disordered persons, diabetics, persons who were in antibiotic therapy. We excluded alcoholics. We excluded smokers, non-physical exercise persons.

This study was conducted on 150 male persons and having age more than 35 years and not more than 60 years of age. Blood samples were taken from normotensive, primary hypertensive and secondary hypertensive people; 50 male persons are considered in each group. Blood is taken before meal at afternoon. Height and weight were measured with the subject in light clothes without shoes and Body Mass Index (BMI) $(\mathrm{Kg} / \mathrm{m} 2)$ was calculated.

In this study the ESR, CRP and lipid profile [(Twelve hour fasting lipid analysis was done for Serum triglycerides (TG), total cholesterol (TC), High Density Lipoprotein (HDL), Low Density Lipoprotein (LDL)] levels were considered. C-Reactive Protein (CRP), Erythrocyte Sedimentation Rate (ESR) and lipid profile values were taken to evaluate future risk factors in cardiovascular disease. CRP levels were identified by using latex slide test, by using Span Diagnostic Ltd. Company Kit, Gujarat. It is a semiquantitative slide test and a drop of serum is mixed with a drop of CRP reagent. Erythrocyte sedimentation rate levels were identified by performing Westergren's method. It was performed by holding anticoagulant mixed blood in a test tube for hours. Lipid profile was performed through ERBA diagnostics kit and SPAN diagnostics kit.

The subjects considered were interviewed and basic history was taken into consideration. The subjects were given proper precautions and self consent form was taken before drawing blood.

Statistical analysis was done by using Microsoft excel 2010. ANOVA test and T test was done to analyze data. Ethical committee permission was taken. The results of the study were expressed as mean \pm standard deviation. P-value $<0.05$ was considered statistically significant.

\section{RESULTS}

We evaluated total 150 working male. The maximum and minimum age considered for our study is $>35$ years and $<60$ years; 50 persons were considered per each group to perform lipid profile, ESR and CRP in normotensive, primary hypertensive and secondary hypertensive male working people. We observed increase in CRP, ESR, LDL-C, triglycerides, cholesterol levels in secondary hypertensive male people.

There is an increase in CRP, ESR, LDL-C, triglycerides, cholesterol levels as compared with normotensive male and primary hypertension people. HDL-C levels are decreased in secondary hypertensive male people. The MEAN and STDEV of age, BMI, vegetarian/non-vegetarian, systolic blood pressure, diastolic blood pressure, sector of working were plotted in Table 1.

\begin{tabular}{|c|c|c|c|c|}
\hline Sl. No. & $\begin{array}{l}\text { Normotensive } \\
\text { MEAN } \pm \text { STDEV }\end{array}$ & $\begin{array}{c}\text { Primary Hypertensive } \\
\text { MEAN } \pm \text { STDEV }\end{array}$ & $\begin{array}{c}\text { Secondary Hypertensive } \\
\text { MEAN } \pm \text { STDEV }\end{array}$ & $P$ value \\
\hline Age & $42.83 \pm 4.99$ & $48.16 \pm 6.52$ & $47.36 \pm 6.89$ & Not significant \\
\hline BMI & $26.94 \pm 3.02$ & $24.64 \pm 2.37$ & $26.81 \pm 3.22$ & Not significant \\
\hline $\begin{array}{l}\text { Vegetarians/non- } \\
\text { vegetarians }\end{array}$ & $22 / 28$ & $8 / 42$ & $14 / 36$ & \\
\hline Systolic BP & $108.73 \pm 4.85$ & $143.86 \pm 6.94$ & $179.2 \pm 32.54$ & Highly significant \\
\hline Diastolic BP & $70.66 \pm 4.82$ & $102.61 \pm 6.61$ & $128.8 \pm 19.20$ & Highly significant \\
\hline Working sector & $\begin{array}{c}\text { Daily waged labour 18/ } \\
\text { employees32 }\end{array}$ & $\begin{array}{c}\text { Daily waged labour 21/ } \\
\text { employees29 }\end{array}$ & $\begin{array}{c}\text { Daily waged labour 10/ } \\
\text { employees } 40\end{array}$ & \\
\hline Obese & 50 people & 50 people & 50 people & \\
\hline
\end{tabular}


We observed increase in age is one of the risk factor for hypertension and we observed no variation in BMI levels in obese individuals, even though person's age advances. In our study, we observed higher number of non-vegetarian employees have hypertension than the vegetarians and daily waged labour count.

\begin{tabular}{|c|c|c|c|c|c|}
\hline Sl. No & TEST & $\begin{array}{c}\text { Normotensive } \\
\text { MEAN } \pm \text { SD }\end{array}$ & $\begin{array}{c}\text { Primary Hypertensive } \\
\text { MEAN } \pm \text { SD }\end{array}$ & Secondary Hypertensive MEAN \pm SD & P-value \\
\hline 1 & CRP & $0.96 \pm 0.32$ & $1.01 \pm 0.35$ & $1.2 \pm 0.40$ & $<0.05$ \\
\hline 2 & ESR & $7.9 \pm 2.07$ & $13.23 \pm 2.82$ & $15.83 \pm 4.42$ & $<0.05$ \\
\hline 3 & \multicolumn{7}{|c|}{ Lipid profile } & $<0.05$ \\
\hline a) & Triglycerides & $198.66 \pm 61.43$ & $212.5 \pm 64.76$ & $276.33 \pm 70.69$ & $<0.05$ \\
\hline b) & cholesterol & $166.93 \pm 23.38$ & $187.46 \pm 46.75$ & $212.2 \pm 39.6$ & $<0.05$ \\
\hline c) & LDL-C & $99.4 \pm 19.42$ & $107.86 \pm 19.51$ & $146.76 \pm 27.27$ & $<0.05$ \\
\hline d) & HDL-C & $50.4 \pm 6.56$ & $40.53 \pm 4.32$ & $35.13 \pm 5.01$ & \\
\hline \multicolumn{7}{|c|}{ Table 2: Comparison of Inflammatory Markers and Lipid Profile Levels in Elderly Male Obese Individuals } \\
\hline
\end{tabular}

There is significant increase in ESR, cholesterol, triglycerides, LDL-C levels in primary \& secondary hypertensive people than the normotensive levels. CRP levels are increased than the normal levels. HDL-C levels decreased less than normal levels in secondary hypertensive obese people.

\begin{tabular}{|c|c|c|c|c|}
\hline Sl. No. & Test & Normotensive & Primary Hypertensive & P-value \\
\hline 1 & CRP & $0.96 \pm 0.32$ & $1.01 \pm 0.35$ & $<0.2$ \\
\hline 2 & ESR & $7.9 \pm 2.07$ & $13.23 \pm 2.82$ & $<0.05$ \\
\hline 3 & Lipid profile & $198.66 \pm 61.43$ & $212.5 \pm 64.76$ & $<0.2$ \\
\hline a) & Triglycerides & $166.93 \pm 23.38$ & $187.46 \pm 46.75$ & $<0.05$ \\
\hline b) & Cholesterol & $99.4 \pm 19.42$ & $107.86 \pm 19.51$ & $<0.05$ \\
\hline c) & LDL-C & $50.4 \pm 6.56$ & $40.53 \pm 4.32$ & $<0.05$ \\
\hline d) & HDL-C & Table 3: Comparison of Inflammatory Markers and Lipid Profile \\
\hline \multicolumn{5}{|r|}{} \\
\end{tabular}

We compared normotensive obese male and essential hypertensive obese male. We recognized brisk increase in ESR, triglycerides, LDL-C levels. We observed no definite change in CRP, HDL-C levels, but there is a increase in CRP levels and decrease in HDL-C levels within normal limits.

\begin{tabular}{|c|c|c|c|c|}
\hline Sl. No. & Test & Primary Hypertensive & Secondary Hypertensive MEAN \pm SD & P-value \\
\hline 1 & CRP & $1.01 \pm 0.35$ & $1.2 \pm 0.40$ & $<0.05$ \\
\hline 2 & ESR & $13.23 \pm 2.82$ & $15.83 \pm 4.42$ & $<0.05$ \\
\hline 3 & Lipid profile & & & $<0.05$ \\
\hline a) & Triglycerides & $212.5 \pm 64.76$ & $276.33 \pm 70.69$ & $<0.05$ \\
\hline b) & Cholesterol & $187.46 \pm 46.75$ & $212.2 \pm 39.6$ & $<0.05$ \\
\hline c) & LDL-C & $107.86 \pm 19.51$ & $146.76 \pm 27.27$ & $<0.05$ \\
\hline d) & HDL-C & $40.53 \pm 4.32$ & $35.13 \pm 5.01$ & \\
\hline \multicolumn{7}{|c|}{ Table 4: Comparison of Inflammatory Markers and Lipid Profile Levels in } \\
\\
\hline \multicolumn{7}{|c|}{ Primary Hypertensive and Secondary Hypertensive Male Obese Individuals. } \\
\hline
\end{tabular}

When we compared primary hypertensive people with secondary hypertensive people, there is an increase in triglycerides, cholesterol and LDL-C levels and decrease in HDL-C levels less than normal levels. There is an increase in inflammatory marker levels.

\section{DISCUSSION}

In our study we observed increase in CRP, ESR and lipid profile levels. Many studies indicated CRP levels increase with age. According to Hokama and Nakamura, serum CRP levels increase progressively with age. ${ }^{18}$ Increasing age, smoking, symptoms of chronic bronchitis, Helicobacter pylori and chlamydia infection and basal metabolic index were all associated with raised levels of CRP, so we excluded smokers and disordered persons. Alcohol consumption is associated with a decreased probability of elevated CRP supporting an anti-inflammatory mechanism, by which a moderate alcohol use might protect against cardiovascular death.19 Blood markers of inflammation are strongly associated with chronic disease in the older populations and inflammation has been implicated in pathology of several cardiovascular and metabolic diseases. Evidence also supports that exercise has anti-inflammatory effects, so we excluded who were prone to exercise everyday. Higher levels of habitual physical activity are associated with low levels of CRP.20 Low grade inflammation and elevation of CRP has also been associated with obesity, high blood pressure and diabetes. In our study we proved obesity is not only the reason for increase in blood pressure. Our study is supported by many similar studies. Obese people have normal triglycerides and LDL-C levels and there is marked increase in triglycerides, LDL-C levels in nonobese individuals. ${ }^{21}$ In our study, we observed employees are having primary or secondary hypertension than the daily waged workers. We observed statistically highly significant relation between normal lipid profile levels and secondary hypertensive people lipid profile levels. 
We observed statistically highly significant relation between normal ESR levels and secondary hypertensive people ESR levels. We observed primary increase and significant relation between CRP levels in normotensive and secondary hypertensive male obese individuals. We observe marked increase in triglycerides, LDL-C and ESR levels in normotensive and in essential hypertensive male obese individuals. We did not observe definite change in CRP levels in normotensive and in essential hypertensive male obese individuals.

\section{CONCLUSION}

We observed an increase in ESR, LDL-C, cholesterol and triglycerides levels in secondary hypertensive male individuals and definite fall in HDL-C levels in secondary hypertensive male individuals, but not in essential hypertensive individuals. We have also observed increase in CRP levels. Since change in lipid profile and increase in ESR and CRP levels lead to cardiovascular complications in near future, thorough examination and early intervention either by lifestyle modifications or therapeutic applications may be required among hypertensive obese individuals.

\section{BIBLIOGRAPHY}

1. Bautista LE, et al. Association between C-reactive protein \& hypertension in healthy middle aged men \& women. Coron artery dis 2004;15.

2. Ridker PM, et al. C-reactive protein \& other markers of inflammation in the prediction of cardiovascular disease in women. N engl J med 2000;342:836-843.

3. Hansen HS, Larsen ML. Hypertension \& Hyperlipidemia. Ugeskr Laeger. 2009 jun 8;171(24):2028-30.

4. Olskaker JS. The erythrocyte sedimentation rate. J emerg med 2002;15(6):713.

5. Prasad K. C-reactive protein - lowering agents. Cardiovasc drug rev 2006;24(1):33-50.

6. Cotron, Kumar, Collins. Blood vessels. In: Robbins pathologic basis of disease. $6^{\text {th }}$ ed. Philadelphia: Saunders 2000:506.

7. Kotsis V, Stabouli S, et al. Mechanism of obesity-induced hypertension res 2010 may;33(5):386-93.

8. Chae CU, Lee RT. Blood pressure \& inflammation in apparently healthy men. Hypertension 2001;38:399-403.
9. Norman M, Kaplan. Clinical hypertension: 9th ed. Lippincott and Wilkins. USA 2006;15-16.

10. Malik R, Pirzado ZA, et al. Study of lipid profile, blood pressure \& blood glucose in rural population. Pak J Med Res 1995;34:152-5.

11. Prineas RJ, Gillium RF. The Minneapolis children blood pressure study: standards of blood pressure for children's blood pressure. Hypertension 1980 Jul-aug; 2: 118-23.

12. Robertson Strong. Development of atherosclerosis in dogs with hypercholesterolemia \& chronic hypertension. Lab invest 1968;2:243.

13. Mendall MA, et al.: C-reactive protein \& its relation to cardiovascular disease risk factors: a population based cross sectional study 2001;312:1061-1065.

14. Dana E King, Arch GM. Dietary magnesium \& CRP levels. American college of nutrition 2005; volume 24(no.3): 166-171.

15. Park EJ. Effect of dietary carbohydrate on triglyceride metabolism in humans. Journal of nutrition 2001;131,2772s-4s.

16. Price D, Ramachandran S, et al. Observed changes in lipid profile \& calculated risk in patients given dietary advice in primary care. British journal of general practitioners 2000;50:712-5.

17. Yu-poth, Zhao G, Etherton T, et al. Effects of the national cholesterol education programs step-I \& step-II dietary intervention programs on cardiovascular disease risk factors: a meta analysis. American journal of clinical nutrition 1999;69:632-46.

18. Hokoma Y, Nakamura RM. CRP: Current status \& future prospectus. Journal of clinic laboratory analysis-1. 1987;15-27.

19. Scott H Stewart. Relation between alcohol consumption $\&$ CRP levels in the adult us population. $3^{\text {rd }}$ national health \& nutritional examination survey 2002; volume 15(no.6): 437-442.

20. Gleeson M, M Fartion BK, Flymn MG. Exercise \& toll like receptors. Excr Immunol 2006; rev12: 34-53.

21. Sarkar D, Latif SA, et al.: Studies on serum lipid profile in hypertensive patient. Mymensingh med J. 2007 jan; 16(1): 70-6. 